\title{
Efeito da (in)atividade física nas queixas de insônia, humor e qualidade de vida de pacientes com hipertensão arterial
}

http://dx.doi.org/10.11606/1807-5509202000030385

\author{
Alana Weikert Neves* \\ Marcos Gonçalves Santana* \\ Katryne Holanda Silva* \\ Giselle Soares Passos*
}

*Universidade Federal de Goiás, Jataí, GO, Brasil.

\section{Resumo}

0 objetivo do presente estudo foi comparar as queixas de insônia, o perfil de humor e a qualidade de vida de pacientes com hipertensão arterial fisicamente ativos e inativos; e identificar possiveis correlações entre insônia, humor, qualidade de vida e nível de atividade física. Participaram do estudo 71 pacientes, com idade > 40 anos, que foram alocados em dois grupos: grupo fisicamente ativo (GFA, $n=36$ ) e grupo fisicamente inativo ( $\mathrm{GFI}, \mathrm{n}=35)$. Os instrumentos utilizados foram: Índice de Gravidade de Insônia, Profile of Mood States, SF-36/Pesquisa em Saúde e Nivel de Atividade Física Habitual. Na comparação entre os grupos, observou-se diferença significante na gravidade das queixas de insônia (GFA: $2,8 \pm 3,4 ; G F I$ : $5,6 \pm 5,6, p<0,05$ ), sintomas de depressão (GFA: $4,4 \pm 5,0 ; G F I: 8,1 \pm 7,0, p<0,05$ ) e as variáveis de qualidade de vida: capacidade funcional (GFA: $84,0 \pm 21,1$; GFI: 71,0 $\pm 23,7$ ) e vitalidade (GFA: $74,9 \pm 20,4$; GFI: $63,1 \pm 22,2)$, além de correlação significante entre as queixas de insônia, o humor e a qualidade de vida. Diante desses resultados podemos concluir que praticar exercícios físicos regularmente pode resultar em redução nas queixas de insônia e depressão, e melhorar alguns aspectos da qualidade de vida de pacientes com hipertensão arterial.

Palavras-Chave: Exercício; Insônia; Depressão; Ansiedade; Hipertensão Arterial.

\section{Introdução}

A insônia é o distúrbio do sono mais comum em todo mundo. Aproximadamente $1 / 3$ da população mundial apresenta queixas de insônia, sendo cerca de $15 \%$ deste total diagnosticado com insônia crônica ${ }^{1}$. Dados de um estudo epidemiológico realizado no Brasil (São Paulo) demonstram que cerca de 45\% da população adulta/idosa tem queixas subjetivas de insônia, 32\% possuem queixas objetivas e 15\% diagnóstico clínico pelo DSM-IV².

Caracterizada pela dificuldade para iniciar ou para manter o sono, pelo despertar precoce ou pelo sono não restaurador, a insônia crônica frequentemente resulta em prejuízos durante o dia, como fadiga excessiva, falta de atenção, concentração e memória, sonolência diurna excessiva e problemas sociais, familiares e ocupacionais ${ }^{3,4}$. Nessa perspectiva, o humor e a qualidade de vida dos pacientes com insônia podem ficar comprometidos ao longo do tempo.
A associação entre os distúrbios do sono e as doenças cardiovasculares (DCV) tem sido amplamente descrita na literatura ${ }^{5-7}$. A apneia obstrutiva do sono (AOS) é o distúrbio que está mais diretamente associado à essas patologias, tendo sido considerada causa secundária, bem como fator de risco para a hipertensão arterial ${ }^{8-12}$. Devulapally, Pongonis e Khayat ${ }^{10}$ a definem como "a nova doença cardiovascular".

Além disso, na última década, tem sido discutida a relação entre a insônia e a incidência de DCV, incluindo hipertensão arterial, doença coronariana e insuficiência cardíaca. Alguns mecanismos têm sido propostos para explicar essa relação, entre eles, a desregulação do eixo hipotálamo-hipófise-adrenal, o aumento da atividade do sistema nervoso simpático, e o aumento do processo inflamatório ${ }^{13,14}$. 
$\mathrm{Na}$ última década, o efeito do exercício físico no tratamento da insônia crônica tem sido bastante investigado, sendo um dos mecanismos propostos para explicar esse efeito, a melhora na função imunológica ${ }^{15}$.

Entre as terapias não farmacológicas propostas pela VII Diretrizes Brasileiras de Hipertensão ${ }^{16}$, como co-adjuvantes ao tratamento medicamentoso, destaca-se a prática regular de exercícios físicos. Cornelissen e FAGARD $^{17}$ investigaram a efetividade do exercício aeróbio na redução da pressão arterial (PA). A redução média (PAsistólica/diastólica) descrita neste estudo foi de aproximadamente 2,4/1,6 $\mathrm{mmHg}$ para indivíduos normotensos e de $6,9 / 4,9 \mathrm{mmHg}$ para os pacientes hipertensos.

\section{Método}

\section{Procedimentos Éticos}

O estudo foi aprovado pelo Comitê de Ética em Pesquisa da Universidade Federal de Goiás - UFG (CEP: 018/14). Os procedimentos experimentais seguiram os princípios da Declaração de Helsinque. Todos os voluntários foram esclarecidos sobre todos os procedimentos, e, concordando em participar, os mesmos leram e assinaram o Termo de Consentimento Livre e Esclarecido (TCLE).

\section{Amostra}

Os 71 participantes do estudo (55 mulheres e 16 homens) foram selecionados nas Unidades de Saúde e Programas de Atendimento aos pacientes com Hipertensão Arterial, da cidade de Jataí, GO.

Foram classificados como fisicamente ativos os pacientes que praticavam atividade física, pelo menos 30 minutos por dia, no mínimo 3 vezes por semana [grupo fisicamente ativo - GFA $(\mathrm{n}=36)]$ e foram considerados inativos aqueles que não praticavam atividade física regular [grupo fisicamente inativo - GFI $(\mathrm{n}=35)$ ].

Os critérios de inclusão foram: (a) idade $>40$ anos; (b) diagnóstico de hipertensão arterial, segundo as VII Diretrizes Brasileiras de Hipertensão ${ }^{16}$. Critério de não inclusão: (1) uso de medicamentos para transtornos psiquiátricos
Considerando-se a relação entre hipertensão arterial e os distúrbios do sono, bem como os efeitos do exercício físico no controle dos níveis pressóricos, acredita-se que hipertensos fisicamente ativos, além de possivelmente apresentar níveis pressóricos mais próximos da normalidade, podem ter menos queixas de má qualidade do sono, menor incidência de distúrbios do sono, melhor perfil de humor, além de melhor qualidade de vida, quando comparados aos inativos. Diante disso, os objetivos deste estudo são (1) comparar as queixas de insônia, o perfil de humor e qualidade de vida de pacientes com hipertensão arterial fisicamente ativos e inativos; (2) identificar possíveis correlações entre insônia, humor, qualidade de vida e nível de atividade física.

ou para distúrbios de sono.

\section{Desenho do Estudo}

Este é um estudo de corte transversal, cujo procedimento experimental foi subdividido em três visitas:

$1^{a}$ Visita ao laboratório: avaliação física, que incluiu: (1) medida da PA; (2) medida da massa corporal e da estatura.

$2^{\text {a }}$ Visita ao laboratório: preenchimento dos instrumentos (auto-avaliação).

$3^{a}$ Visita ao laboratório: entrega do resultado das avaliações aos participantes do estudo.

\section{Descrição dos instrumentos}

\section{Indice de Massa Corporal - IMC}

O IMC foi calculado individualmente $\left(\mathrm{IMC}=\right.$ peso/altura ${ }^{2}$ ), após a mensuração da massa corporal e da estatura dos participantes. A medida da massa corporal foi realizada em balança mecânica (Welmy, Brasil). Essa balança possui capacidade para até $200 \mathrm{Kg}$, com precisão de $100 \mathrm{~g}$. A estatura das participantes foi determinada em um estadiômetro portátil (Sanny, Brasil), com precisão de $1 \mathrm{~mm}$.

\section{Medida da Pressão Arterial (PA)}

Para a medida da pressão arterial, foi utilizado 
um esfigmomanômetro semi-automático digital de braço (OMRON-HEM-431, USA), seguindo os critérios recomendados pela VII Diretrizes Brasileiras de Hipertensão ${ }^{16}$. Tais critérios consistem em: explicar ao paciente todo o procedimento, deixando-o de repouso por 5 minutos em ambiente calmo; esclarecer possíveis dúvidas antes ou após o procedimento, instruindo-o a não conversar durante a medida; certificar-se de que o paciente não está com a bexiga cheia, não praticou exercícios físicos nos últimos 60 minutos, não ingeriu bebidas alcóolicas, café ou alimentos e que não fumou nos últimos 30 minutos. Além disso, deve-se posicionar o paciente sentado, com pés apoiados no chão, pernas descruzadas, dorso relaxado e recostado na cadeira. Quanto ao braço, este deve estar na altura do coração, livre de roupa, apoiado com a palma da mão voltada para cima e com cotovelo ligeiramente fletido.

\section{Indice de Gravidade de Insônia - IGI}

O IGI é um instrumento desenvolvido para avaliar as queixas de insônia. É uma escala de autoavaliação, breve e simples, composta por 7 itens que podem ser classificados de 0 a 4 , sendo o escore final mais baixo $=0$ e mais alto $=28$. Considera-se insônia não significativa escores de $0 \mathrm{a} 7$, limite inferior para insônia de 8 a 14, insônia moderada de 15 a 21 e insônia grave de 22 a $28^{18}$.

\section{Profile of Mood States (POMS)}

O perfil de humor é avaliado por meio de uma escala que varia de 0 a 4 . Este instrumento possui 65 itens, que resultam em 6 domínios: tensãoansiedade; depressão; raiva-hostilidade; vigor; fadiga; confusão mental. $\mathrm{O}$ escore total é derivado da diferença entre os escores da escala vigor e a soma dos escores dados pelas outras sub-escalas. Considera-se normal quando, em um gráfico, a curva se apresenta em forma de "iceberg", na qual a sub-escala vigor fica acima do percentil 50 , enquanto os demais fatores ficam abaixo dele ${ }^{19}$.

\section{SF-36 Pesquisa em Saúde - Medical Outcomes Study (SF-36)}

É um instrumento multidimensional que engloba 8 componentes: capacidade funcional, aspectos físicos, emocionais e sociais, vitalidade, estado geral de saúde, dor e saúde mental. Por avaliar tanto aspectos negativos (enfermidades) como positivos (bem-estar), este questionário tem a função de avaliar, de forma genérica, a qualidade de vida. Os escores de cada componente são ranqueados de 0 a 100, com escores mais altos indicando melhor qualidade de vida ${ }^{20}$.

\section{Nivel de Atividade Física Habitual (NAFH)}

O NAFH é um instrumento de auto-avaliação, que identifica o nível de atividade física habitual ${ }^{21}$. É constituído por 21 itens, divididos em três esferas: (1) nível de atividade física no trabalho; (2) nível de prática esportiva e (3) índice de atividade de lazer. O escore total é obtido por meio da somatória das três esferas, sendo que quanto maior for os escores, maior é o nível de atividade física.

\section{Análise de dados}

Para a análise dos dados foi utilizado o programa STATISTICA® (versão 7.0). Para verificar a normalidade dos dados foi utilizado o teste Kolmogorov Smirnov. Como a maioria das variáveis apresentou distribuição normal ( $p>0,05)$, optou-se por testes paramétricos. Foi utilizado o teste $t$-Student para amostras independentes, para comparação entre os grupos (fisicamente ativo e inativo), e o teste de correlação de Pearson para verificar a associação entre as médias dos diversos domínios dos instrumentos IGI, POMS, SF-36 e NAFH. O nível de significância adotado foi de $\mathrm{p}<0,05$.

\section{Resultados}

\section{Características físicas}

Não foi observada diferença significante no IMC entre os grupos $(p>0,05)$. O GFA apresentou

valores inferiores de pressão arterial sistólica quando comparado ao GFI, mas quanto à pressão arterial diastólica, não foram observadas diferenças entre os grupos (TABELA 1). 
TABELA 1-Características físicas dos grupos fisicamente ativo (GFA) e fisicamente inativo (GFI).

Teste t-Student,

dados apresentados

como média \pm D.P.

IMC: Índice de Mas-

sa Corporal; PAS:

Pressão Arterial Sis-

tólica; PAD: Pressão

Arterial Diastólica.

\begin{tabular}{lccc}
\hline VARIÁVEIS & GFI $(\mathbf{n}=\mathbf{3 5})$ & GFA $(\mathbf{n}=\mathbf{3 6})$ & $\mathbf{p}$ \\
\hline Sexo $(\mathrm{M} / \mathrm{F})$ & $8 / 27$ & $8 / 28$ & - \\
Idade (anos) & $57,5 \pm 10$ & $61,2 \pm 9$ & 0,11 \\
IMC $\left(\mathrm{Kg} / \mathrm{m}^{2}\right)$ & $29,3 \pm 5,1$ & $28,7 \pm 4,5$ & 0,64 \\
PAS (mmHg) & $135,7 \pm 16,5$ & $126,1 \pm 14,2$ & 0,01 \\
PAD (mmHg) & $74,6 \pm 19,1$ & $74,2 \pm 18,6$ & 0,92 \\
\hline
\end{tabular}

Como se pode observar na TABELA 2, houve diferença significante nos escores Índice de Atividade Esportiva, Índice de Atividade de Lazer e escore absoluto do Nível de Atividade Física Habitual (NAFH) entre os grupos.
Quanto às análises de correlação, foi observada correlação negativa do Índice de Atividade Esportiva (IAE) avaliado pelo instrumento NAFH com os valores de pressão arterial sistólica $(\mathrm{r}=-0,42 ; \mathrm{p}<0,05)$.

Teste $t$-Student, dados apresentados como média \pm D.P. GFI: grupo fisicamente inativo; GFA: grupo fisicamente ativo.

\begin{tabular}{lccc}
\multicolumn{2}{l}{ TABELA 2-Dados obtidos pelo instrumento Nível de Atividade Física Habitual (NAFH). } \\
\hline VARIÁVEIS (escores) & GFI (n=35) & GFA (n=36) & p \\
\hline Índice Ocupacional & $2,6 \pm 0,6$ & $2,6 \pm 0,5$ & 0,88 \\
Índice de Atividade Esportiva & $1,8 \pm 0,5$ & $2,6 \pm 0,6$ & $<0,01$ \\
Índice de Atividade de Lazer & $2,0 \pm 0,6$ & $2,6 \pm 0,7$ & $<0,01$ \\
Total absoluto & $6,3 \pm 1,4$ & $7,8 \pm 1,0$ & $<0,01$ \\
\hline
\end{tabular}

\section{Insônia, Perfil de humor e Qualidade de Vida}

Quanto às queixas de insônia, observou-se que cerca de $28 \%$ dos participantes do GFI e $11 \%$ do GFA apresentaram insônia significativa (escore do IGI>7). Na comparação entre os grupos, observou-se diferença significante entre eles $(\mathrm{p}<0,05)$. Em relação ao humor, o GFA apresentou menor escore de depressão quando comparado ao GFI (TABELA 3).

Quanto à qualidade de vida, foi observada diferença significante entre os grupos fisicamente ativo e fisicamente inativo, indicando que o grupo fisicamente ativo apresentou melhor qualidade de vida quando comparado ao fisicamente inativo, no que se refere aos aspectos Capacidade Funcional e Vitalidade (TABELA 4).

TABELA 3-Dados obtidos por meio dos instrumentos Profile of Mood States (POMS) e Índice de Gravidade da Insônia (IGI).

Teste t-Student, dados apresentados como média \pm D.P. GFI: grupo fisicamente inativo; GFA: grupo fisicamente ativo.

\begin{tabular}{lccc}
\hline \multicolumn{1}{c}{ VARIÁVEIS (escores) } & GFI $(\mathrm{n}=\mathbf{3 5})$ & GFA $(\mathrm{n}=\mathbf{3 6})$ & $\mathrm{p}$ \\
\hline POMS & & & \\
Tensão/ansiedade & $8,2 \pm 6,3$ & $6,9 \pm 5,5$ & 0,37 \\
Depressão & $8,1 \pm 7,0$ & $4,4 \pm 5,0$ & 0,01 \\
Raiva/hostilidade & $7,6 \pm 7,1$ & $5,5 \pm 5,4$ & 0,16 \\
Vigor & $19,0 \pm 6,0$ & $20,1 \pm 5,5$ & 0,45 \\
Fadiga & $6,8 \pm 4,8$ & $6,1 \pm 3,8$ & 0,45 \\
Confusão Mental & $2,0 \pm 3,6$ & $1,8 \pm 4,5$ & 0,84 \\
Distúrbio total de humor & $14,3 \pm 24,9$ & $5,4 \pm 21,5$ & 0,11 \\
IGI & $5,6 \pm 5,6$ & $2,8 \pm 3,4$ & 0,01 \\
\hline
\end{tabular}


TABELA 4-Dados obtidos por meio do instrumento SF-36 Pesquisa em Saúde.

\begin{tabular}{lccc}
\hline VARIÁVEIS (escores) & GFI $(\mathbf{n}=\mathbf{3 5})$ & GFA $(\mathbf{n}=\mathbf{3 6})$ & $\mathbf{p}$ \\
\hline Capacidade funcional & $71,0 \pm 23,7$ & $84,0 \pm 21,1$ & 0,02 \\
Aspectos Físicos & $77,1 \pm 35,5$ & $70,1 \pm 43,4$ & 0,46 \\
Dor & $70,5 \pm 26,4$ & $66,4 \pm 29,7$ & 0,53 \\
Estado geral de saúde & $71,3 \pm 16,4$ & $79,0 \pm 17,9$ & 0,06 \\
Vitalidade & $63,1 \pm 22,2$ & $74,9 \pm 20,4$ & 0,02 \\
Aspectos Sociais & $88,6 \pm 21,9$ & $88,9 \pm 22,3$ & 0,95 \\
Aspectos Emocionais & $74,3 \pm 36,2$ & $77,8 \pm 38,2$ & 0,69 \\
Saúde mental & $77,7 \pm 16,9$ & $81,7 \pm 17,2$ & 0,33 \\
\hline
\end{tabular}

Teste $t$-Student,

dados apresentados como média \pm D.P. GFI: grupo fisicamente inativo; GFA: grupo fisicamente

\section{Correlaçóes}

\section{Nivel de Atividade Física e Qualidade de Vida}

Observou-se correlação positiva do Índice de Atividade Esportiva avaliado pelo NAFH com a Vitalidade avaliada pelo SF-36 $(r=0,27$; $\mathrm{p}<0,05)$.

Sono, Humor e Qualidade de Vida

Foram observadas correlações positivas da gravidade das queixas de insônia avaliada pelo Índice de Gravidade da Insônia (IGI) com os seguintes domínios do POMS: Tensão-Ansiedade $(\mathrm{r}=0,38 ; \mathrm{p}<0,05)$; Raiva-Hostilidade $(\mathrm{r}=0,37$; $\mathrm{p}<0,05)$; Fadiga $(\mathrm{r}=0,39 ; \mathrm{p}<0,05)$; Confusão Mental $(r=0,30 ; p<0,05)$; Depressão $(r=0,47$; $\mathrm{p}<0,05)$ e Distúrbio Total de Humor $(\mathrm{r}=0,53$; $\mathrm{p}<0,05)$. As duas últimas correlaçóes citadas estão graficamente representadas na FIGURA 1. Em adição, observou-se correlação negativa da gravidade das queixas de insônia com o domínio Vigor avaliado pelo POMS $(r=-0,32 ; \mathrm{p}<0,05)$.
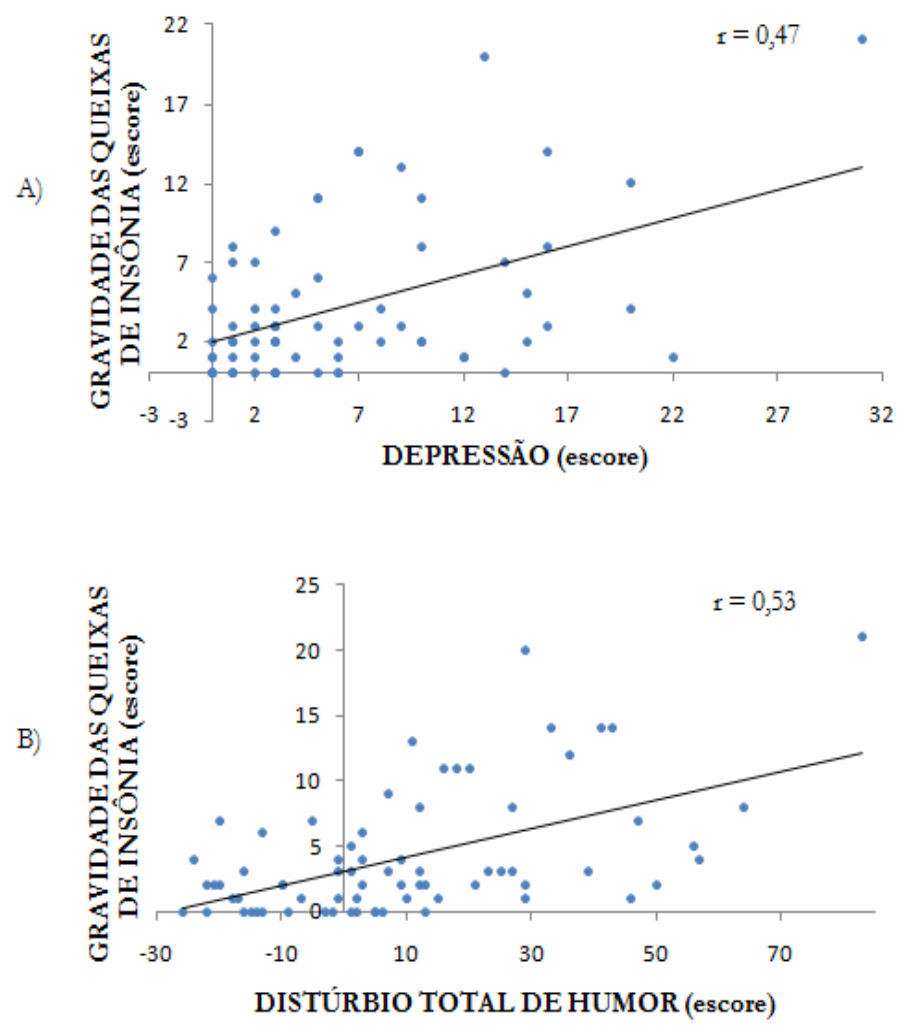

FIGURA 1-A) Correlação entre a Gravidade das Queixas de Insônia e a Depressão. B) Correlação entre a Gravidade das Queixas de Insônia e o Distúrbio Total de Humor. Teste de correlação de Pearson, p<0,05. 
Além disso, observou-se correlação negativa da gravidade das queixas de insônia com os escores: Capacidade Funcional ( $\mathrm{r}=-0,27 ; \mathrm{p}<0,05)$; Aspectos Físicos $(\mathrm{r}=-0,29 ; \mathrm{p}<0,05)$; Dor $(\mathrm{r}=$ -0,26; $\mathrm{p}<0,05)$; Estado Geral de Saúde ( $\mathrm{r}=-0,30$; $\mathrm{p}<0,05)$; Vitalidade $(\mathrm{r}=-0,36 ; \mathrm{p}<0,05)$; Aspectos Sociais $(r=-0,38 ; p<0,05)$; Aspectos Emocionais ( $r$ $=-0,31 ; \mathrm{p}<0,05)$ e Saúde Mental $(\mathrm{r}=-0,29 ; \mathrm{p}<0,05)$ avaliados pelo SF-36.

Todos os domínios do POMS correlacionaramse significantemente com os escores do SF-36, que avalia a qualidade de vida. Entre as correlaçôes, podemos destacar a correlação negativa do domínio Tensão-Ansiedade com os Aspectos Físicos ( $\mathrm{r}=$ 0,26; $\mathrm{p}<0,05)$; a Vitalidade $(\mathrm{r}=-0,32 ; \mathrm{p}<0,05)$; os Aspectos Sociais $(\mathrm{r}=-0,26 ; \mathrm{p}<0,05)$; os Aspectos Emocionais $(r=-0,24 ; p<0,05)$ e a Saúde Mental $(r$ $=-0,37 ; \mathrm{p}<0,05)$ avaliados pelo SF-36. O domínio Depressão também correlacionou negativamente como Estado Geral de Saúde $(\mathrm{r}=-0,26 ; \mathrm{p}<0,05)$; com a Vitalidade $(\mathrm{r}=-0,46 ; \mathrm{p}<0,05)$; com os Aspectos Sociais $(r=-0,30 ; p<0,05)$; com Aspectos Emocionais $(r=-0,24 ; p<0,05)$ e com a Saúde Mental $(r=-0,45 ; \mathrm{p}<0,05)$ avaliados pelo SF-36.

\section{Discussão}

O objetivo do presente estudo foi comparar queixas de insônia, perfil de humor e qualidade de vida de pacientes com hipertensão arterial fisicamente ativos e inativos, e identificar possíveis correlaçóes entre essas variáveis.

Em relação à gravidade das queixas de insônia, ambos os grupos apresentaram insônia não significativa (escore do IGI < 7). No entanto, os participantes fisicamente ativos apresentaram valores significantemente inferiores quando comparados aos fisicamente inativos, indicando menos queixas de insônia neste grupo. O efeito positivo do exercício físico na insônia está bem estabelecido na literatura ${ }^{15}$. Algumas hipóteses têm sido estudadas para explicar essa relação, entre elas os efeitos ansiolítico e antidepressivo do exercício físico. Segundo Youngstedt ${ }^{22}$, o efeito do exercício físico na redução da ansiedade é uma justificativa plausível para explicar a melhora na qualidade do sono, visto que a ansiedade é um dos marcadores de insônia. Portanto, um estímulo capaz de reduzir os níveis de ansiedade, poderia promover o sono. Já a teoria antidepressiva sugere que a redução da depressão decorrente da prática de exercício físico poderia, em longo prazo, promover o sono. Segundo Passos et al. ${ }^{15}$ a insônia pode ser um fator de risco para o desenvolvimento da depressão, assim como pode ser desencadeada como conseqüência deste transtorno psiquiátrico.

$\mathrm{O}$ presente estudo apresentou resultados que corroboram com as afirmaçôes anteriores, visto que houve diferença significativa no domínio depressão entre o grupo fisicamente ativo e fisicamente inativo. Além disso, observou-se correlação positiva do escore obtido pelo Índice de Gravidade da
Insônia com os domínios Depressão e TensãoAnsiedade obtidos pelo POMS, indicando que quanto mais graves foram as queixas de insônia, piores estavam os índices de depressão e tensãoansiedade dos participantes. Além disso, observouse correlação negativa da gravidade das queixas de insônia com o nível de vigor dos participantes, indicando que quanto menor era a gravidade das queixas de insônia, mais alto era o nível de vigor. Houve também correlação positiva da gravidade das queixas de insônia com os níveis de RaivaHostilidade, Fadiga, Confusão Mental e Distúrbio Total de Humor, indicando que quanto menor foi gravidade das queixas de insônia, menores foram os níveis destes aspectos negativos do humor.

No presente estudo observou-se ainda correlação positiva da gravidade das queixas de insônia avaliada com todos os escores de qualidade de vida, indicando que, quanto maior a gravidade das queixas de insônia dos participantes, pior era a sua qualidade de vida. LeBLANC et al. ${ }^{23}$, relacionando as variáveis de qualidade de vida com a insônia crônica, mostrou que indivíduos insones tinham menor qualidade de vida e maiores índices de depressão, ansiedade, neuroticismo, extraversão, predisposição à excitação, percepção de estresse e enfrentamento emocional.

Müller e Guimarāes ${ }^{24}$, em uma revisão de literatura, apontam que há um consenso entre os autores de que a insônia compromete a qualidade de vida. Segundo Passos et al. ${ }^{25}$, os efeitos negativos dos distúrbios do sono sobre a qualidade de vida estão bem documentados na literatura. Em seu estudo, os autores observaram que, após a prática de exercício aeróbio durante seis meses, 
pacientes com insônia crônica apresentaram melhora na qualidade de vida, particularmente na dimensão emocional.

Além disso, houve correlação negativa dos níveis de depressão, tensão-ansiedade, raiva-hostilidade, fadiga, confusão mental e distúrbio total de humor com a maioria das variáveis indicadoras de qualidade de vida. Essas correlações indicam que, quanto mais negativo foi o estado de humor, pior estava a qualidade de vida dos participantes do estudo. Segundo Peluso e Guerra de Andrade ${ }^{26}$ e Kahlbaugh et al. ${ }^{27}$, evidências demonstram que o exercício físico melhora o estado de humor ou ajuda a manter seus níveis, podendo ser utilizado na prevenção e no tratamento dos transtornos do humor, de forma a promover melhora da qualidade de vida.

Os escores de qualidade de vida observados no presente estudo são semelhantes aos observados em outros estudos com pacientes hipertensos ${ }^{28,29}$. Embora ambos sejam relativamente baixos, o GFA apresentou maior capacidade funcional e vitalidade, quando comparado ao GFI. Além disso, observou-se correlação positiva do índice de atividade esportiva identificado no NAFH e o escore vitalidade do SF-36, indicando que quanto maior o índice de atividade esportiva dos participantes, maior era a vitalidade dos mesmos. Evidências de correlação entre qualidade de vida e nível de atividade física foram previamente descritas por Pucci et al. ${ }^{30}$.

Apesar de ambos os grupos apresentarem a pressão arterial controlada, o grupo fisicamente inativo apresentou valor médio considerado limítrofe $(135 / 74 \mathrm{mmHg})$, enquanto o grupo fisicamente ativo apresentou valor médio mais próximo da normalidade $(126 / 74 \mathrm{mmHg}$ ), considerando os padrōes estabelecidos pela VII Diretrizes Brasileiras de Hipertensão Arterial ${ }^{16}$.

Observou-se também correlação negativa entre o Índice de Atividade Esportiva e a pressão arterial sistólica dos participantes, indicando que, quanto maior o índice de atividade esportiva dos participantes, menor foi o valor da pressão arterial sistólica. A diferença observada na pressão arterial sistólica entre os grupos, foi em média $9 \mathrm{mmHg}$. O efeito dos exercícios aeróbios, sejam agudos ou crônicos, no sistema cardiovascular, tais como redução da PA no repouso e em cargas submáximas de esforço, está bem estabelecido na literatura. Uma recente metanálise avaliou a efetividade do exercício aeróbio na redução da PA. A redução média (PAS/ PAD) descrita neste estudo foi de aproximadamente 2,4/1,6 $\mathrm{mmHg}$ para indivíduos normotensos e de $6,9 / 4,9 \mathrm{mmHg}$ para os pacientes hipertensos ${ }^{17}$.

Podemos apontar duas limitaçôes no presente estudo. A primeira delas é o tipo de estudo. Estudos transversais não permitem estabelecer uma relação de causa e efeito. Portanto, não podemos inferir que o exercício físico foi responsável pelos benefícios descritos neste estudo, somente podemos assumir que os fisicamente ativos diferenciaram-se dos inativos. A segunda limitação é a dificuldade no controle do medicamento usado no tratamento da hipertensão arterial. Não foi possível controlar o medicamento, devido às especificidades de cada caso. Há uma grande variedade de medicamentos usados no tratamento da hipertensão arterial, e eles são prescritos considerando-se as co-morbidades e, principalmente a adaptação e tolerância de cada paciente.

Diante dos resultados deste estudo podemos concluir que pacientes com hipertensão arterial que praticam exercícios físicos, apresentam menor gravidade das queixas de insônia, depressão, e melhor qualidade de vida (no que se refere aos aspectos capacidade funcional e vitalidade), quando comparados aos fisicamente inativos. Além disso, quanto menor foi a gravidade das queixas de insônia dos participantes, melhor estava o perfil de humor e a qualidade de vida dos pacientes.

Pesquisas futuras, de caráter experimental, que investiguem o efeito do exercício físico, de longo prazo, no sono e humor de pacientes com hipertensão arterial, são necessárias para fortalecer a conclusão de que a prática regular de exercício físico pode resultar em menor gravidade das queixas de insônia e, consequentemente, melhoras no humor e na qualidade de vida de pacientes com hipertensão arterial.

\section{Agradecimentos}

Agradecemos aos pacientes que participaram voluntariamente desse estudo. 


\begin{abstract}
Effect of physical (in)activity on sleep, mood and quality of life in patients with hypertension

The aim of the present study was to compare insomnia complaints, mood profile and quality of life of physically active and inactive hypertension patients; and to identify possible correlations between insomnia, mood, quality of life and physical activity level. Seventy-one patients, aged $>40$ years, were alocated in two groups: ACTIVE $(n=36)$ and INACTIVE $(n=35)$. The instruments used were: Insomnia Severity Index, Profile of Mood States, SF-36/Medical Outcomes Study. Significant difference was observed in the severity of insomnia complaints (ACTIVE: $2,8 \pm 3,4$; INACTIVE: $5,6 \pm 5,6, p<0,05$ ), symptoms of depression (ACTIVE: $4,4 \pm 5,0$; INACTIVE: $8,1 \pm 7,0, p<0,05)$ and quality of life: functional capacity (ACTIVE: $84,0 \pm 21,1$; INACTIVE: $71,0 \pm 23,7$ ) and vitality (ACTIVE: $74,9 \pm 20,4$; INACTIVE: $63,1 \pm 22,2$ ) and significant correlation of insomnia with mood and quality of life. Considering these results, we can conclude exercise training could promote reduction of insomnia complaints and depression, and improve some aspects of quality of life in patients with hypertension.
\end{abstract}

KEYWORDS: Exercise; Insomnia; Depression; Quality of Life; Hypertension.

\title{
Referências
}

1. Ohayon MM. Prevalence and comorbidity of sleep disorders in general population. La Revue du praticien. 2007;57(14):1521-8.

2. Castro LS, Poyares D, Leger D, Bittencourt L, Tufik S. Objective prevalence of insomnia in the Sao Paulo, Brazil epidemiologic sleep study. Ann Neurol. 2013;74(4):537-46.

3. American Psychiatric Association. Diagnostic and Statistical Manual of Mental Disorders (DSM-V). Washington: American Psychiatric Association; 2013.

4. American Academy of Sleep Medicine. International classification of sleep disorders (ICSD-3). Darien: AASM Resource Library; 2014.

5. Greenland P, Knoll MD, Stamler J, Neaton JD, Dyer AR, Garside DB, et al. Major risk factors as antecedents of fatal and nonfatal coronary heart disease events. JAMA. 2003;290(7):891-7.

6. Sabanayagam C, Shankar A. Sleep duration and cardiovascular disease: results from the National Health Interview Survey. Sleep. 2010;33(8):1037-42.

7. Vgontzas AN, Liao D, Bixler EO, Chrousos GP, Vela-Bueno A. Insomnia with objective short sleep duration is associated with a high risk for hypertension. Sleep. 2009;32(4):491-7.

8. Pepin JL, Borel AL, Tamisier R, Baguet JP, Levy P, Dauvilliers Y. Hypertension and sleep: overview of a tight relationship. Sleep Med Rev. 2014;18(6):509-519.

9. Bagai K. Obstructive sleep apnea, stroke, and cardiovascular diseases. Neurologist. 2010;16(6):329-39.

10. Devulapally K, Pongonis R Jr, Khayat R. OSA: the new cardiovascular disease: part II: Overview of cardiovascular diseases associated with obstructive sleep apnea. Heart Fail Rev. 2009;14(3):155-64.

11. Levy P, Tamisier R, Arnaud C, Monneret D, Baguet JP, Stanke-Labesque F, et al. Sleep deprivation, sleep apnea and cardiovascular diseases. Front Biosci (Elite Ed). 2012;4:2007-21.

12. Rivas M, Ratra A, Nugent K. Obstructive sleep apnea and its effects on cardiovascular diseases: a narrative review. Anatol J Cardiol. 2016;15(11):944-50.

13. Sofi F, Cesari F, Casini A, Macchi C, Abbate R, Gensini GF. Insomnia and risk of cardiovascular disease: a meta-analysis. Eur J Prev Cardiol. 2014;21(1):57-64.

14. Javaheri S, Redline S. Insomnia and risk of cardiovascular disease. Chest. 2017;152(2):435-444.

15. Passos GS, Poyares DL, Santana MG, Tufik S, Mello MT. Is exercise an alternative treatment for chronic insomnia? Clinics. 2012;67(6):653-60.

16. Sociedade Brasileira de Cardiologia. VII Diretrizes Brasileiras de Hipertensão. Arq Bras Cardiol. 2016;107(3Supl.3):183. 
17. Cornelissen VA, Fagard RH. Effects of endurance training on blood pressure, blood pressure-regulating mechanisms, and cardiovascular risk factors. Hypertension. 2005;46(4):667-75.

18. Bastien CH, Vallieres A, Morin CM. Validation of the Insomnia Severity Index as an outcome measure for insomnia research. Sleep Medicine. 2001;2(4):297-307.

19. McNair DM, Lorr M, Droppleman LF. Profile of mood state manual. San Diego (CA): Educational and Industrial Testing Service; 1971.

20. da Mota Falcão D, Ciconelli RM, Ferraz MB. Translation and cultural adaptation of quality of life questionnaires: an evaluation of methodology. J Rheumatol. 2003;30(2):379-85.

21. Baecke JA, Burema J, Frijters JE. A short questionnaire for the measurement of habitual physical activity in epidemiological studies. Am J Clin Nutr. 1982;36(5):936-52.

22. Youngstedt SD. Effects of exercise on sleep. Clin Sports Med. 2005;24(2):355-65.

23. LeBlanc M, Beaulieu-Bonneau S, Merette C, Savard J, Ivers H, Morin CM. Psychological and health-related quality of life factors associated with insomnia in a population-based sample. J Psychosom Res. 2007;63(2):157-66.

24. Müller MR, Guimarães SS. Impacto dos transtornos do sono sobre o funcionamento diário e a qualidade de vida. Estud psicol. 2007;24(4):519-28.

25. Passos GS, Poyares D, Santana MG, D'Aurea CV, Youngstedt SD, Tufik S, et al. Effects of moderate aerobic exercise training on chronic primary insomnia. Sleep Med. 2011;12(10):1018-27.

26. Peluso MA, Guerra de Andrade LH. Physical activity and mental health: the association between exercise and mood. Clinics. 2005;60(1):61-70.

27. Kahlbaugh PE, Sperandio AJ, Carlson AL, Hauselt J. Effects of playing Wii on well-being in the elderly: Physical activity, loneliness, and mood. Act Adapt Aging. 2011;35(4):331-44.

28. Carvalho MA, Silva IB, Ramos SB, Coelho LF, Goncalves ID, Figueiredo Neto JA. Quality of life of hypertensive patients and comparison of two instruments of HRQOL measure. Arq Bras Cardiol. 2012;98(5):442-51.

29. Youssef RM, Moubarak, II, Kamel MI. Factors affecting the quality of life of hypertensive patients. East Mediterr Health J. 2005;11(1-2):109-18.

30. Pucci GCMF, Rech CR, Fermino RC, Reis RS. Associação entre atividade física e qualidade de vida em adultos. Rev Saúde Públ. 2012;46(1):166-79.

ENDEREÇO DE CORRESPONDÊNCIA:

Giselle Soares Passos

BR 364, km 195, nº 3800 Jataí - GO - BRASIL

CEP: 75801-615.

E-mail: passos.gs@gmail.com; alanawn.edf@gmail.com
Submetido: 22/06/2017

Revisado: 11/10/2017

Aceito: 13/11/2017 